\title{
OPERATOR-OPERATOR PADA HIMPUNAN KABUR HESITANT BERNILAI INTERVAL
}

\author{
AWANDA AMELIA MARON, YUDIANTRI ASDI \\ Program Studi Matematika, \\ Fakultas Matematika dan Ilmu Pengetahuan Alam, Universitas Andalas, \\ Kampus UNAND Limau Manis Padang, Indonesia. \\ email : ameliawanda446@gmail.com
}

Diterima 9 Maret 2019 Direvisi 7 April 2019 Dipublikasikan 7 Mei 2019

\begin{abstract}
Abstrak. Chen dan Xu memperkenalkan tentang relasi preference hesitant bernilai interval dalam proses pengambilan keputusan kelompok(Group Decision Making/GDM) [2]. Pada proses $G D M$ digunakan operator-operator untuk mengumpulkan informasi Interval-valued Hesitant Fuzzy Set (IVHFS) [2]. Konsep himpunan kabur hesitant bernilai interval banyak digunakan pada teori pengambilan keputusan. akan tetapi pada penelitian ini hanya dibatasi kajian aljabar yaitu dikaji tentang sifat-sifat operasi pada elemen kabur hesitant bernilai interval dan bentuk operator-operator pada IVHFS. Operasi ring sum, ring product, irisan dan gabungan pada elemen kabur hesitant bernilai interval memenuhi sifat-sifat aljabar yaitu sifat komutatif, sifat asosiatif, sifat distributif. Bentuk operator-operator pada himpunan kabur hesitant bernilai interval yaitu operator GIVHFWA, GIVHFWG dan operator GIVHFOWA, GIVHFOWG.
\end{abstract}

Kata Kunci: Himpunan kabur hesitant bernilai interval, Sifat-sifat operasi, Operator

\section{Pendahuluan}

Di dalam kehidupan sehari-hari terdapat beberapa permasalahan yang berbedabeda, dimana salah satunya melibatkan sifat manusia. Akibatnya, setiap permasalahan harus diperhatikan untuk mendapatkan solusi yang memuaskan. Permasalahan yang sering ditemukan adalah permasalahan mengenai ketidakpastian. Ketidakpastian dapat diperhatikan untuk mengambil suatu keputusan pada perbedaan pendapat. Pengambilan keputusan tidak bisa hanya dengan menggunakan metode yang klasik, karena beberapa dari kasus mengandung unsur ketidakpastian dan keragu-raguan.

Pada tahun 1965, himpunan kabur (Fuzzy Set/FS) pertama kali diperkenalkan oleh Prof.L.A. Zadeh yang mengkaji tentang derajat keanggotaan dari suatu elemen, yang mana derajat keanggotaan tersebut dinyatakan dengan nilai tunggal dalam interval [0,1] [9]. Pada tahun 2009 Torra dan Narukawa memperkenalkan konsep dari himpunan kabur hesitant (Hesitant Fuzzy Set/HFS) [5]. Kemudian, Chen dan Xu (2013) memperkenalkan tentang relasi preference hesitant bernilai interval dalam proses $G D M$ [2]. Pada proses $G D M$, relasi preferensi hesitant merupakan alat yang 
populer oleh pembuat keputusan (Decision Makers/DMs) dalam membandingkan suatu keputusan. Pada teori ini digunakan operator-operator untuk mengumpulkan informasi Interval-valued Hesitant Fuzzy Set/IVHFS [2]. Konsep himpunan kabur hesitant bernilai interval banyak digunakan pada teori pengambilan keputusan. Akan tetapi pada penelitian ini hanya dibatasi kajian aljabar yaitu akan dikaji tentang sifat-sifat operasi pada elemen kabur hesitant dan bentuk operator-operator pada IVHFS.

Dalam hal ini diperoleh sifat-sifat operasi pada elemen kabur hesitant bernilai interval, yaitu komutatif pada ring sum, ring product, distributif, irisan dan gabungan. Bentuk operator-operator pada himpunan kabur hesitant bernilai interval yaitu operator Generalized Interval-Valued Hesitant Fuzzy Weight Averaging (GIVHFWA), Generalized Interval-Valued Hesitant Fuzzy Weight Geometric (GIVHFWG) dan operator Generalized Interval-Valued Hesitant Fuzzy Ordered Weight Averaging (GIVHFOWA), Generalized Interval-Valued Hesitant Fuzzy Ordered Weight Geometric (GIVHFOWG).

\section{Landasan Teori}

\subsection{Himpunan Kabur (Fuzzy Set)}

Definisi 2.1. [9] Misalkan $X$ adalah himpunan semesta, maka himpunan kabur $A$ atas $X$ didefinisikan sebagai:

$$
A=\left\{\left(x, \mu_{A}(x)\right) \mid x \in X\right\},
$$

dimana $\mu_{A}: X \rightarrow[0,1]$ dan $\mu_{A}(x)$ disebut derajat keanggotaan dari $X$ atas $A$.

\subsection{Himpunan Kabur Hesitant (Hesitant Fuzzy Set)}

Definisi 2.2. [3] Misalkan $X$ adalah himpunan semesta, maka himpunan kabur hesitant $F$ atas $X$ didefinisikan sebagai:

$$
F=\left\{\left\langle x, h_{F}(x)\right\rangle \mid x \in X\right\},
$$

dimana $h_{F}(x)$ merupakan himpunan dari beberapa nilai yang berbeda pada interval $[0,1]$ yang menotasikan derajat keanggotaan HFS di $x \in X$ pada $F$. Selanjutnya derajat keanggotaan pada HFS disebut dengan Hesitant Fuzzy Element (HFE).

Definisi 2.3. [4,5] Misalkan terdapat tiga Hesitant Fuzzy Element (HFE) $h, h_{1}, h_{2}$. Komplemen, gabungan dan irisan didefinisikan sebagai berikut:

(1) $h^{c}=\bigcup_{\gamma \in h}\{1-\gamma\}$;

(2) $h_{1} \cup h_{2}=\bigcup_{\gamma_{1} \in h_{1}, \gamma_{2} \in h_{2}}$ $\max \left\{\gamma_{1}, \gamma_{2}\right\}$

(3) $h_{1} \cap h_{2}=\bigcup_{\gamma_{1} \in h_{1}, \gamma_{2} \in h_{2}}$ $\min \left\{\gamma_{1}, \gamma_{2}\right\}$.

Formula operasi pada tiga Hesitant Fuzzy Element (HFE) $h, h_{1}, h_{2}$ diberikan oleh Xia dan $\mathrm{Xu}[7]$. 
Definisi 2.4. [7] Misalkan $h, h_{1}, h_{2}$ adalah tiga Hesitant Fuzzy Element (HFE), didefinisikan sebagai berikut:

(1) $h^{\lambda}=\bigcup_{\gamma \in h}\left\{\gamma^{\lambda}\right\}, \lambda>0$

(2) $\lambda h=\bigcup_{\gamma \in h}\left\{1-(1-\gamma)^{\lambda}\right\}, \lambda>0$;

(3) $h_{1} \oplus h_{2}=\bigcup_{\gamma_{1} \in h_{1}, \gamma_{2} \in h_{2}}\left\{\gamma_{1}+\gamma_{2}-\gamma_{1} \gamma_{2}\right\}$;

(4) $h_{1} \otimes h_{2}=\bigcup_{\gamma_{1} \in h_{1}, \gamma_{2} \in h_{2}}\left\{\gamma_{1} \gamma_{2}\right\}$.

Definisi 2.5. [1] Misalkan $S$ adalah suatu himpunan bagian yang tak kosong di $\mathbb{R}$, atau $S \subseteq \mathbb{R}$ :

(a) Himpunan $S$ dikatakan terbatas di atas, jika terdapat $u \in \mathbb{R}$ sedemikian sehingga $s \leqslant u$ untuk setiap $s \in S$ dan u disebut batas atas dari $S$.

(b) Himpunan $S$ dikatakan terbatas di bawah, jika terdapat $w \in \mathbb{R}$ sedemikian sehingga $s \geqslant w$ untuk setiap $s \in S$ dan $w$ disebut batas bawah dari $S$.

Definisi 2.6. [1] Misalkan $S$ adalah suatu himpunan bagian yang tak kosong di $\mathbb{R}$, atau $S \subseteq \mathbb{R}$.

(a) Jika $S$ terbatas di atas, maka $u \in \mathbb{R}$ dikatakan supremum (atau batas atas terkecil) dari $S$ jika memenuhi kondisi berikut:

(1) u adalah batas atas dari $S$, dan

(2) jika $v \in \mathbb{R}$ adalah sebarang batas atas dari $S$, maka $u \leqslant v$.

(b) Jika $S$ terbatas di bawah, maka $w \in \mathbb{R}$ dikatakan infimum (atau batas bawah terbesar) dari $S$ jika memenuhi kondisi berikut:

(1) w adalah batas bawah dari $S$, dan

(2) jika $t \in \mathbb{R}$ adalah sebarang batas bawah dari $S$, maka $u \geqslant v$.

\subsection{Himpunan Kabur Hesitant Bernilai Interval (Interval-Valued Hesitant Fuzzy Set)}

Definisi 2.7. [2] Misalkan $X=\left\{x_{1}, x_{2}, \cdots, x_{n}\right\}$ adalah suatu himpunan semesta. Suatu himpunan kabur hesitant bernilai interval $\tilde{A}$ atas $X$ dapat dinyatakan sebagai

$$
\tilde{A}=\left\{\left\langle x_{i}, \tilde{h}_{\tilde{A}}\left(x_{i}\right)\right\rangle \mid x_{i} \in X, i=1,2, \cdots, n\right\}
$$

dimana $\tilde{h}_{\tilde{A}}\left(x_{i}\right)$ merupakan himpunan dari beberapa subinterval yang berbeda pada interval $[0,1]$ yang menotasikan derajat keanggotaan IVHFS di $x \in X$ pada $\tilde{A}$. Derajat keanggotaan dapat ditulis sebagai:

$$
\tilde{h}_{\tilde{A}}\left(x_{i}\right)=\left\{\tilde{\gamma} \mid \tilde{\gamma} \in \tilde{h}_{\tilde{A}}\left(x_{i}\right)\right\},
$$

dimana $\tilde{\gamma}=\left[\tilde{\gamma}^{L}, \tilde{\gamma}^{U}\right]$ adalah suatu bilangan interval, $\tilde{\gamma}^{L}$ adalah batas bawah terbesar dari $\tilde{\gamma}$ dan $\tilde{\gamma}^{U}$ adalah batas atas terkecil dari $\tilde{\gamma}$.

Definisi 2.8. [8] Misalkan $\tilde{a}=\left[\tilde{a}^{L}, \tilde{a}^{U}\right]$ dan $\tilde{b}=\left[\tilde{b}^{L}, \tilde{b}^{U}\right]$ dua bilangan interval dan $\lambda \geqslant 0$, didefinisikan sebagai berikut.

(1) $\tilde{a}=\tilde{b}$ jika $\tilde{a}^{L}=\tilde{b}^{L}$ dan $\tilde{a}^{U}=\tilde{b}^{U}$; 
(2) $\tilde{a}+\tilde{b}=\left[\tilde{a}^{L}+\tilde{b}^{L}, \tilde{a}^{U}+\tilde{b}^{U}\right]$;

(3) $\lambda \tilde{a}=\left[\lambda \tilde{a}^{L}, \lambda \tilde{a}^{U}\right]$ khususnya $\lambda \tilde{a}=0$, jika $\lambda=0$.

Fungsi skor untuk elemen kabur hesitant bernilai interval (Interval-Valued Hesitant Fuzzy Set/IVHFE) didefinisikan sebagai berikut:

Definisi 2.9. [2] Untuk suatu Interval-Valued Hesitant Fuzzy Element (IVHFE), $S(\tilde{h})=\frac{1}{l_{\tilde{h}}} \sum_{\tilde{\gamma} \in \tilde{h}} \tilde{\gamma}=\frac{1}{l_{\tilde{h}}}\left[\sum_{\tilde{\gamma} \in \tilde{h}} \tilde{\gamma}^{L}, \sum_{\tilde{\gamma} \in \tilde{h}} \tilde{\gamma}^{U}\right]$ disebut suatu fungsi skor dari $\tilde{h}$ dan $l_{\tilde{h}}$ adalah banyak bilangan interval di $\tilde{h}$ dan $S(\tilde{h})$ suatu bilangan interval yang berada pada selang $[0,1]$. Untuk dua IVHFE $\tilde{h}_{1}$ dan $\tilde{h}_{2}$, jika $S\left(\tilde{h}_{1}\right) \geqslant S\left(\tilde{h}_{2}\right)$ maka dinotasikan $\tilde{h}_{1} \geqslant \tilde{h}_{2}$.

Beberapa operasi pada Interval-Valued Hesitant Fuzzy Element (IVHFE), didefinisikan sebagai berikut.

Definisi 2.10. [2] Misalkan $\tilde{h}, \tilde{h}_{1}$ dan $\tilde{h}_{2}$ merupakan tiga Interval-Valued Hesitant Fuzzy Element (IVHFE), didefinisikan sebagai berikut.

(1) $\tilde{h}^{c}=\left\{\left[1-\tilde{\gamma}^{U}, 1-\tilde{\gamma}^{L}\right] \mid \tilde{\gamma} \in \tilde{h}\right\}$;

(2) $\tilde{h}_{1} \cup \tilde{h}_{2}=\left\{\left[\max \left\{\tilde{\gamma}_{1}^{L}, \tilde{\gamma}_{2}^{L}\right\}, \max \left\{\tilde{\gamma}_{1}^{U}, \tilde{\gamma}_{2}^{U}\right\}\right] \mid \tilde{\gamma}_{1} \in \tilde{h}_{1}, \tilde{\gamma}_{2} \in \tilde{h}_{2}\right\}$;

(3) $\tilde{h}_{1} \cap \tilde{h}_{2}=\left\{\left[\min \left\{\tilde{\gamma}_{1}^{L}, \tilde{\gamma}_{2}^{L}\right\}, \min \left\{\tilde{\gamma}_{1}^{U}, \tilde{\gamma}_{2}^{U}\right\}\right] \mid \tilde{\gamma}_{1} \in \tilde{h}_{1}, \tilde{\gamma}_{2} \in \tilde{h}_{2}\right\}$;

(4) $\tilde{h}^{\lambda}=\left\{\left[\left(\tilde{\gamma}^{L}\right)^{\lambda},\left(\tilde{\gamma}^{U}\right)^{\lambda}\right] \mid \tilde{\gamma} \in \tilde{h}\right\}$;

(5) $\lambda \tilde{h}=\left\{\left[1-\left(1-\tilde{\gamma}^{L}\right)^{\lambda}, 1-\left(1-\tilde{\gamma}^{U}\right)^{\lambda}\right] \mid \tilde{\gamma} \in \tilde{h}\right\}, \lambda>0$;

(6) $\tilde{h}_{1} \oplus \tilde{h}_{2}=\left\{\left[\tilde{\gamma}_{1}^{L}+\tilde{\gamma}_{2}^{L}-\left(\tilde{\gamma}_{1}^{L}\right)\left(\tilde{\gamma}_{2}^{L}\right), \tilde{\gamma}_{1}^{U}+\tilde{\gamma}_{2}^{U}-\left(\tilde{\gamma}_{1}^{U}\right)\left(\tilde{\gamma}_{2}^{U}\right)\right] \mid \tilde{\gamma}_{1} \in \tilde{h}_{1}, \tilde{\gamma}_{2} \in \tilde{h}_{2}\right\}$;

(7) $\tilde{h}_{1} \otimes \tilde{h}_{2}=\left\{\left[\left(\tilde{\gamma}_{1}^{L}\right)\left(\tilde{\gamma}_{2}^{L}\right),\left(\tilde{\gamma}_{1}^{U}\right)\left(\tilde{\gamma}_{2}^{U}\right)\right] \mid \tilde{\gamma}_{1} \in \tilde{h}_{1}, \tilde{\gamma}_{2} \in \tilde{h}_{2}\right\}$.

Saat $\tilde{\gamma}^{L}=\tilde{\gamma}^{U}$ maka operasi pada Definisi 2.10 menjadi operasi pada Definisi 2.3 dan Definisi 2.4.

Lema 2.11. [7] Misalkan $x_{j}>0, \lambda_{j}>0, j=1,2, \cdots, n$ dan $\sum_{j=1}^{n} \lambda_{j}=1$, maka:

$$
\prod_{j=1}^{n} x_{j}^{\lambda_{j}} \leq \sum_{j=1}^{n} \lambda_{j} x_{j}
$$

Selanjutnya, $\prod_{j=1}^{n} x_{j}^{\lambda_{j}}=\sum_{j=1}^{n} \lambda_{j} x_{j}$ berlaku jika dan hanya jika $x_{1}=x_{2}=\cdots=$ $x_{n}$.

\section{Pembahasan}

Pada bab ini akan dibahas tentang sifat-sifat operasi pada elemen kabur hesitant bernilai interval (Interval-Valued Hesitant Fuzzy Set/IVHFE) dan bentuk operatoroperator pada himpunan kabur hesitant bernilai interval (Interval-Valued Hesitant Fuzzy Set/IVHFS).

\subsection{Sifat-Sifat Operasi pada Elemen Kabur Hesitant Bernilai Interval (IVHFE)}

Teorema 3.1. [2] Misalkan $\tilde{h}, \tilde{h}_{1}$ dan $\tilde{h}_{2}$ adalah tiga Interval-Valued Hesitant Fuzzy Element (IVHFE), maka: 
(1) $\tilde{h}_{1} \oplus \tilde{h}_{2}=\tilde{h}_{2} \oplus \tilde{h}_{1}$;

(2) $\tilde{h}_{1} \otimes \tilde{h}_{2}=\tilde{h}_{2} \otimes \tilde{h}_{1}$;

(3) $\lambda\left(\tilde{h}_{1} \oplus \tilde{h}_{2}\right)=\lambda \tilde{h}_{1} \oplus \lambda \tilde{h}_{2}, \lambda>0$;

(4) $\left(\tilde{h}_{1} \otimes \tilde{h}_{2}\right)^{\lambda}=\left(\tilde{h}_{1}\right)^{\lambda} \otimes\left(\tilde{h}_{2}\right)^{\lambda}, \lambda>0$;

(5) $\lambda_{1} \tilde{h} \oplus \lambda_{2} \tilde{h}=\left(\lambda_{1}+\lambda_{2}\right) \tilde{h}, \lambda_{1}, \lambda_{2}>0$;

(6) $\tilde{h}^{\lambda_{1}} \otimes \tilde{h}^{\lambda_{2}}=\tilde{h}^{\left(\lambda_{1}+\lambda_{2}\right)}, \lambda_{1}, \lambda_{2}>0$.

Teorema 3.2. [2] Misalkan $\tilde{h}, \tilde{h}_{1}$ dan $\tilde{h}_{2}$ adalah tiga Interval-Valued Hesitant Fuzzy Element (IVHFE), maka:

(1) $\tilde{h}_{1}^{c} \cup \tilde{h}_{2}^{c}=\left(\tilde{h}_{1} \cap \tilde{h}_{2}\right)^{c}$;

(2) $\tilde{h}_{1}^{c} \cap \tilde{h}_{2}^{c}=\left(\tilde{h}_{1} \cup \tilde{h}_{2}\right)^{c}$;

(3) $\left(\tilde{h}^{c}\right)^{\lambda}=(\lambda \tilde{h})^{c}$;

(4) $\lambda\left(\tilde{h}^{c}\right)=\left(\tilde{h}^{\lambda}\right)^{c}$;

(5) $\tilde{h}_{1}^{c} \oplus \tilde{h}_{2}^{c}=\left(\tilde{h}_{1} \otimes \tilde{h}_{2}\right)^{c}$;

(6) $\tilde{h}_{1}^{c} \otimes \tilde{h}_{2}^{c}=\left(\tilde{h}_{1} \oplus \tilde{h}_{2}\right)^{c}$.

\subsection{Operator-operator pada Himpunan Kabur Hesitant Bernilai Interval}

Pada bagian ini akan diuraikan bentuk operator-operator lain pada himpunan kabur hesitant bernilai interval (Interval-Valued Hesitant Fuzzy Set/IVH-FS).

Definisi 3.3. [2] Misalkan diketahui suatu Interval-Valued Hesitant Fuzzy Set (IVHFS) $\tilde{H}$ dan $\tilde{h}_{j}$ adalah suatu Interval-Valued Hesitant Fuzzy Element (IVHFE) dari $\tilde{H}$ dan $\boldsymbol{w}=\left(w_{1}, w_{2}, \cdots, w_{n}\right)^{T}$ adalah vektor bobot dari $\tilde{h}_{j}$, dimana $j=$ $1,2, \cdots, n$ dengan $w_{j} \in[0,1], \sum_{j=1}^{n} w_{j}=1$ dan $\lambda>0$ dengan pemetaan dari $\tilde{H}^{n}$ ke $\tilde{H}$, maka:

(1) Operator Interval-Valued Hesitant Fuzzy Weight Averaging (IVHFWA) dengan pemetaan:

$$
\begin{aligned}
& \operatorname{IVHFWA} A\left(\tilde{h}_{1}, \tilde{h}_{2}, \cdots, \tilde{h}_{n}\right)=\oplus_{j=1}^{n}\left(w_{j} \tilde{h}_{j}\right), \\
& =\left\{\left[1-\prod_{j=1}^{n}\left(1-\tilde{\gamma}_{j}^{L}\right)^{w_{j}}, 1-\prod_{j=1}^{n}\left(1-\tilde{\gamma}_{j}^{U}\right)^{w_{j}}\right] \mid \tilde{\gamma}_{1} \in \tilde{h}_{1}, \tilde{\gamma}_{2} \in \tilde{h}_{2}, \cdots, \tilde{\gamma}_{n} \in \tilde{h}_{n}\right\}
\end{aligned}
$$

(2) Operator Interval-Valued Hesitant Fuzzy Weight Geometric (IVHFWG) dengan pemetaan:

$$
\begin{aligned}
& \operatorname{IVHFWG}\left(\tilde{h}_{1}, \tilde{h}_{2}, \cdots, \tilde{h}_{n}\right)=\otimes_{j=1}^{n}\left(\tilde{h}_{j}\right)^{w_{j}}, \\
& =\left\{\left[\prod_{j=1}^{n}\left(\tilde{\gamma}_{j}^{L}\right)^{w_{j}}, \prod_{j=1}^{n}\left(\tilde{\gamma}_{j}^{U}\right)^{w_{j}}\right] \mid \tilde{\gamma}_{1} \in \tilde{h}_{1}, \tilde{\gamma}_{2} \in \tilde{h}_{2}, \cdots, \tilde{\gamma}_{n} \in \tilde{h}_{n}\right\}
\end{aligned}
$$

(3) Operator Generalized Interval-Valued Hesitant Fuzzy Weight Averaging (GIVH- 
FWA) dengan pemetaan:

$$
\begin{aligned}
& \operatorname{GIVHFWA}\left(\tilde{h}_{1}, \tilde{h}_{2}, \cdots, \tilde{h}_{n}\right)=\left(\oplus_{j=1}^{n}\left(w_{j} \tilde{h}_{j}^{\lambda}\right)\right)^{\frac{1}{\lambda}} \\
& =\left\{\left[\left(1-\prod_{j=1}^{n}\left(1-\left(\tilde{\gamma}_{j}^{L}\right)^{\lambda}\right)^{w_{j}}\right)^{\frac{1}{\lambda}},\left(1-\prod_{j=1}^{n}\left(1-\left(\tilde{\gamma}_{j}^{U}\right)^{\lambda}\right)^{w_{j}}\right)^{\frac{1}{\lambda}}\right]\right. \\
& \left.\mid \tilde{\gamma}_{1} \in \tilde{h}_{1}, \tilde{\gamma}_{2} \in \tilde{h}_{2}, \cdots, \tilde{\gamma}_{n} \in \tilde{h}_{n}\right\}
\end{aligned}
$$

(4) Operator Generalized Interval-Valued Hesitant Fuzzy Weight Geometric ( $G I V H F W G$ ) dengan pemetaan

$$
\begin{aligned}
& \operatorname{GIVHFWG}\left(\tilde{h}_{1}, \tilde{h}_{2}, \cdots, \tilde{h}_{n}\right)=\frac{1}{\lambda}\left(\otimes_{j=1}^{n}\left(\lambda \tilde{h}_{j}\right)^{w_{j}}\right) \\
& =\left\{\left[1-\left(1-\prod_{j=1}^{n}\left(1-\left(1-\tilde{\gamma}_{j}^{L}\right)^{\lambda}\right)^{w_{j}}\right)^{\frac{1}{\lambda}}, 1-\left(1-\prod_{j=1}^{n}\left(\tilde{\gamma}_{j}^{U}\right)^{\lambda w_{j}}\right)^{\frac{1}{\lambda}}\right]\right. \\
& \left.\mid \tilde{\gamma}_{1} \in \tilde{h}_{1}, \tilde{\gamma}_{2} \in \tilde{h}_{2}, \cdots, \tilde{\gamma}_{n} \in \tilde{h}_{n}\right\}
\end{aligned}
$$

Jika $\lambda=1$, maka operator GIVHFWA menjadi operator IVHFWA dan operator GIVHFWG menjadi operator IVHFWG. Jika $w=\left(\frac{1}{n}, \frac{1}{n}, \cdots, \frac{1}{n}\right)^{T}$ maka operator IVHFWA menjadi operator IVHFA dan operator IVHFWG menjadi operator IVHFG. Secara khusus didefinisikan sebagai berikut:

(1) Operator IVHFA:

$$
\begin{aligned}
& \operatorname{IVHFA}\left(\tilde{h}_{1}, \tilde{h}_{2}, \cdots, \tilde{h}_{n}\right)=\oplus_{j=1}^{n}\left(\frac{1}{n} \tilde{h}_{j}\right) \\
= & \left\{\left[1-\prod_{j=1}^{n}\left(1-\tilde{\gamma}_{j}^{L}\right)^{\frac{1}{n}}, 1-\prod_{j=1}^{n}\left(1-\tilde{\gamma}_{j}^{U}\right)^{\frac{1}{n}}\right] \mid \tilde{\gamma}_{1} \in \tilde{h}_{1}, \tilde{\gamma}_{2} \in \tilde{h}_{2}, \cdots, \tilde{\gamma}_{n} \in \tilde{h}_{n}\right\}
\end{aligned}
$$

(2) Operator IVHFG:

$$
\begin{aligned}
& \operatorname{IVHFG}\left(\tilde{h}_{1}, \tilde{h}_{2}, \cdots, \tilde{h}_{n}\right)=\otimes_{j=1}^{n}\left(\tilde{h}_{j}\right)^{\frac{1}{n}} \\
& =\left\{\left[\prod_{j=1}^{n}\left(\tilde{\gamma}_{j}^{L} \frac{1}{n}, \prod_{j=1}^{n}\left(\tilde{\gamma}_{j}^{U}\right)^{\frac{1}{n}}\right] \mid \tilde{\gamma}_{1} \in \tilde{h}_{1}, \tilde{\gamma}_{2} \in \tilde{h}_{2}, \cdots, \tilde{\gamma}_{n} \in \tilde{h}_{n}\right\}\right.
\end{aligned}
$$

Contoh 3.4. Misalkan

$$
\begin{aligned}
\tilde{h_{1}} & =\{[0.1,0.3],[0.4,0.5]\} \\
\tilde{h_{2}} & =\{[0.3,0.4],[0.4,0.6],[0.5,0.7]\}
\end{aligned}
$$

merupakan dua IVHFE dan $w=(0.2,0.8)^{T}$ merupakan vektor bobot dari $\tilde{h_{1}}$ dan $\tilde{h_{2}}$. Dengan menggunakan Definisi 3.3 maka: 
(1) Untuk $\lambda=1$,

$$
\begin{aligned}
& G I V H F W A_{1}\left(\tilde{h_{1}}, \tilde{h_{2}}\right)=I V H F W A\left(\tilde{h_{1}}, \tilde{h_{2}}\right)=\oplus_{j=1}^{2}\left(w_{j} \tilde{h}_{j}\right) \\
= & \left\{\left[1-\prod_{j=1}^{2}\left(1-\tilde{\gamma}_{j}^{L}\right)^{w_{j}}, 1-\prod_{j=1}^{2}\left(1-\tilde{\gamma}_{j}^{U}\right)^{w_{j}}\right] \mid \tilde{\gamma}_{1} \in \tilde{h}_{1}, \tilde{\gamma}_{2} \in \tilde{h}_{2}\right\} \\
= & \{[0.2639,0.3812],[0.3493,0.5526],[0.4376,0.6677],[0.3213,0.4215],[0.4,0.5817],[0.4814,0.6677]\} \\
& G I V H F W G_{1}\left(\tilde{h_{1}}, \tilde{h_{2}}\right)=I V H F W G\left(\tilde{h}_{1}, \tilde{h_{2}}\right)=\otimes_{j=1}^{2} \tilde{h}_{j}^{w_{j}} \\
= & \left\{\left[\prod_{j=1}^{2}\left(\tilde{\gamma}_{j}^{L}\right)^{W_{j}}, \prod_{j=1}^{2}\left(\tilde{\gamma}_{j}^{U}\right)^{w_{j}}\right] \mid \tilde{\gamma}_{1} \in \tilde{h}_{1}, \tilde{\gamma}_{2} \in \tilde{h}_{2}\right\} \\
= & \{[0.2408,0.3776],[0.3031,0.5223],[0.3624,0.5909],[0.3178,0.4183],[0.4,0.5785],[0.4782,0.6544]\}
\end{aligned}
$$

(2) Untuk $\lambda=4$

$$
\begin{aligned}
& G I V H F W A_{4}\left(\tilde{h_{1}}, \tilde{h_{2}}\right)=\left(\oplus_{j=1}^{2}\left(w_{j} \tilde{h}_{j}^{4}\right)\right)^{\frac{1}{4}} \\
= & \left\{\left[\left(1-\prod_{j=1}^{2}\left(1-\left(\tilde{\gamma}_{j}^{L}\right)^{4}\right)^{w_{j}}\right)^{\frac{1}{4}},\left(1-\prod_{j=1}^{2}\left(1-\left(\tilde{\gamma}_{j}^{U}\right)^{4}\right)^{w_{j}}\right)^{\frac{1}{4}}\right] \mid \tilde{\gamma}_{1} \in \tilde{h}_{1}, \tilde{\gamma}_{2} \in \tilde{h}_{2}\right\} \\
= & \{[0.2840,0.3857],[0.3786,0.5713],[0.4737,0.6675],[0.3284,0.4265],[0.4,0.5843],[0.4848,0.6749]\} \\
& G I V H F W G_{4}\left(\tilde{h_{1}}, \tilde{h_{2}}\right)=\frac{1}{4}\left(\otimes_{j=1}^{2}\left(4 \tilde{h}_{j}\right)^{w_{j}}\right) \\
= & \left\{\left[1-\left(1-\prod_{j=1}^{2}\left(1-\left(1-\tilde{\gamma}_{j}^{L}\right)^{4}\right)^{w_{j}}\right)^{\frac{1}{4}}, 1-\left(1-\prod_{j=1}^{2}\left(1-\left(1-\tilde{\gamma}_{j}^{U}\right)^{4}\right)^{w_{j}}\right)^{\frac{1}{4}}\right] \mid \tilde{\gamma}_{1} \in \tilde{h}_{1}, \tilde{\gamma}_{2} \in \tilde{h}_{2}\right\} \\
= & \{[0.23,0.3747],[0.2744,0.4804],[0.3053,0.5060],[0.3158,0.4157],[0.4,0.5735],[0.4744,0.6276]\} .
\end{aligned}
$$

Sehingga diperoleh:

$G I V H F W G_{4}\left(\tilde{h_{1}}, \tilde{h_{2}}\right) \leq \operatorname{IVHFWG}\left(\tilde{h_{1}}, \tilde{h_{2}}\right) \leq I V H F W A\left(\tilde{h_{1}}, \tilde{h_{2}}\right) \leq \operatorname{GIVHFW}\left(\tilde{A_{4}}\left(\tilde{h_{1}}, \tilde{h_{2}}\right)\right.$.

Akan dibuktikan secara umum sebagai berikut.

Teorema 3.5. [2] Misalkan $\tilde{h}_{j}$ adalah suatu Interval-Valued Hesitant Fuzzy Element (IVHFE) dengan $j=1,2, \cdots, n . w=\left(w_{1}, w_{2}, \cdots, w_{n}\right)^{T}$ adalah suatu vektor bobot dari $\tilde{h}_{j}$ dengan $w_{j} \in[0,1]$ dan $\sum_{j=1}^{n} w_{j}=1$, maka:

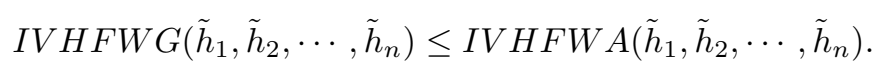

Teorema 3.6. [2] Misalkan $\tilde{h}_{j}$ adalah suatu Interval-Valued Hesitant Fuzzy Element (IVHFE). Misalkan untuk $j=1,2, \cdots, n$, vektor $w=\left(w_{1}, w_{2}, \cdots, w_{n}\right)^{T}$ adalah suatu vektor bobot dari $\tilde{h}_{j}$ dengan $w_{j} \in[0,1]$ dan $\sum_{j=1}^{n} w_{j}=1, \lambda>0$, maka:

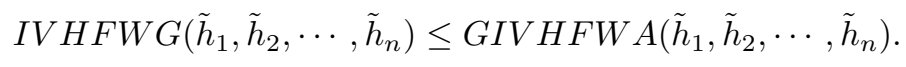


Teorema 3.7. [2] Misalkan $\tilde{h}_{j}$ adalah suatu Interval-Valued Hesitant Fuzzy Element (IVHFE) dengan $j=1,2, \cdots, n . w=\left(w_{1}, w_{2}, \cdots, w_{n}\right)^{T}$ adalah suatu vektor bobot dengan $w_{j} \in[0,1]$ dan $\sum_{j=1}^{n} w_{j}=1, \lambda>0$, maka:

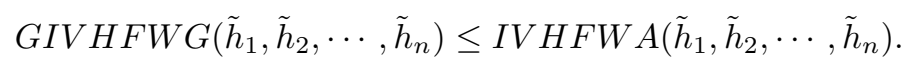

Selanjutnya operator orde bobot rata-rata (Ordered Weight Averaging/OWA) dan operator orde bobot geometris (Ordered Weight Geometric/OWG) diperkenalkan oleh Yager. Operator OWA dan OWG tersusun dalam data yang berurutan dari yang lebih rendah dan dihubungkan dengan suatu vektor, yaitu vektor yang bersesuaian $\left(\omega_{i}\right)$. Pada Definisi 3.8 akan diperkenalkan operator OWA dan OWG untuk informasi himpunan kabur hesitant bernilai interval.

Definisi 3.8. [2] Misalkan diketahui suatu Interval-Valued Hesitant Fuzzy Set (IVHFS) $\tilde{H} . \tilde{h}_{j}$ adalah suatu Interval-Valued Hesitant Fuzzy Element (IVHFE) dari $\tilde{H}$ dengan $j=1,2, \cdots, n, \tilde{h}_{\sigma(j)}$ terbesar ke-j dari $\tilde{h}_{j}, \omega=\left(\omega_{1}, \omega_{2}, \cdots, \omega_{n}\right)^{T}$ adalah suatu vektor yang bersesuaian sedemikian sehingga $w_{j} \in[0,1]$ dan $\sum_{j=1}^{n} w_{j}=1$, $\lambda>0$ dengan pemetaan dari $\tilde{H}^{n}$ ke $\tilde{H}$, maka:

(1) Operator Interval-Valued Hesitant Fuzzy Ordered Weight Averaging (IVHFOWA)

$$
\begin{aligned}
& \operatorname{IVHFOWA}\left(\tilde{h}_{1}, \tilde{h}_{2}, \cdots, \tilde{h}_{n}\right)=\oplus_{j=1}^{n}\left(\omega_{j} \tilde{h}_{\sigma(j)}\right) \\
= & \left\{\left[1-\prod_{j=1}^{n}\left(1-\tilde{\gamma}_{\sigma(j)}^{L}\right)^{\omega(j)}, 1-\prod_{j=1}^{n}\left(1-\tilde{\gamma}_{\sigma(j)}^{U}\right)^{\omega(j)}\right] \mid \tilde{\gamma}_{\sigma(1)} \in \tilde{h}_{\sigma(1)}, \tilde{\gamma}_{\sigma(2)} \in \tilde{h}_{\sigma(2)}, \cdots, \tilde{\gamma}_{\sigma(n)} \in \tilde{h}_{\sigma(n)}\right\}
\end{aligned}
$$

(2) Operator Interval-Valued Hesitant Fuzzy Ordered Weight Geometric (IVHFOWG)

$$
\begin{aligned}
& \operatorname{IVHFOWG}\left(\tilde{h}_{1}, \tilde{h}_{2}, \cdots, \tilde{h}_{n}\right)=\otimes_{j=1}^{n}\left(\tilde{h}_{\sigma(j)}\right)^{\omega_{j}} \\
= & \left\{\left[\prod_{j=1}^{n}\left(\tilde{\gamma}_{\sigma(j)}^{L}\right)^{\omega_{j}}, \prod_{j=1}^{n}\left(\tilde{\gamma}_{\sigma(j)}^{U}\right)^{\omega_{j}}\right] \mid \tilde{\gamma}_{\sigma(1)} \in \tilde{h}_{\sigma(1)}, \tilde{\gamma}_{\sigma(2)} \in \tilde{h}_{\sigma(2)}, \cdots, \tilde{\gamma}_{\sigma(n)} \in \tilde{h}_{\sigma(n)}\right\}
\end{aligned}
$$

(3) Operator Generalized Interval-Valued Hesitant Fuzzy Weight Ordered Averaging (GIVHFOWA)

$$
\begin{aligned}
& \operatorname{GIVHFOWA}\left(\tilde{h}_{1}, \tilde{h}_{2}, \cdots, \tilde{h}_{n}\right)=\left(\oplus_{j=1}^{n} \omega_{j} \tilde{h}_{\sigma(j)}^{\lambda}\right)^{\frac{1}{\lambda}} \\
= & \left\{\left[\left(1-\prod_{j=1}^{n}\left(1-\left(\tilde{\gamma}_{\sigma(j)}^{L}\right)^{\lambda}\right)^{\omega_{j}}\right)^{\frac{1}{\lambda}},\left(1-\prod_{j=1}^{n}\left(1-\left(\tilde{\gamma}_{\sigma(j)}^{U}\right)^{\lambda}\right)^{\omega_{j}}\right)^{\frac{1}{\lambda}}\right]\right. \\
& \left.\mid \tilde{\gamma}_{\sigma(1)} \in \tilde{h}_{\sigma(1)}, \tilde{\gamma}_{\sigma(2)} \in \tilde{h}_{\sigma(2)}, \cdots, \tilde{\gamma}_{\sigma(n)} \in \tilde{h}_{\sigma(n)}\right\}
\end{aligned}
$$

(4) Operator Generalized Interval-Valued Hesitant Fuzzy Ordered Weight Geomet- 


$$
\begin{aligned}
\text { ric } & (\text { GIVHFOWG) } \\
& \text { GIVHFOWG( } \left.\tilde{h}_{1}, \tilde{h}_{2}, \cdots, \tilde{h}_{n}\right)=\frac{1}{\lambda}\left(\otimes_{j=1}^{n}\left(\lambda \tilde{h}_{\sigma(j)}\right)^{\omega_{j}}\right) \\
= & \left\{\left[1-\left(1-\prod_{j=1}^{n}\left(1-\left(1-\tilde{\gamma}_{\sigma(j)}^{L}\right)^{\lambda}\right)^{\omega_{j}}\right) \frac{1}{\lambda}, 1-\left(1-\prod_{j=1}^{n}\left(1-\left(1-\tilde{\gamma}_{\sigma(j)}^{U}\right)^{\lambda}\right)^{\omega_{j}}\right) \frac{1}{\lambda}\right]\right. \\
& \left.\mid \gamma_{\sigma(1)} \in \tilde{h}_{\sigma(1)}, \tilde{\gamma}_{\sigma(2)} \in \tilde{h}_{\sigma(2)}, \cdots, \tilde{\gamma}_{\sigma(n)} \in \tilde{h}_{\sigma(n)}\right\}
\end{aligned}
$$

Jika $\lambda=1$, maka operator GIVHFOWA menjadi operator IVHFOWA dan operator GIVHFOWG menjadi operator IVHFOWG. Jika $\omega=\left(\frac{1}{n}, \frac{1}{n}, \cdots, \frac{1}{n}\right)^{T}$ maka operator IVHFOWA menjadi operator IVHFA dan IVHFOWG menjadi operator IVHFG.

\section{Kesimpulan}

Operasi irisan, gabungan, ring sum dan ring product pada himpunan kabur hesitant bernilai interval (IVHFS memenuhi sifat-sifat aljabar yaitu sifat komutatif, sifat asosiatif, sifat distributif. Selain itu, operator-operator pada himpunan kabur hesitant bernilai interval (IVHFS adalah operator GIVHFWA, GIVHFWG dan operator GIVHFOWA, GIVHFOWG.

\section{Ucapan Terima kasih}

Penulis mengucapkan terima kasih kepada Ibu Nova Noliza Bakar, M.Si, Bapak Prof. Dr. I Made Arnawa dan bapak Zulakmal, M.Si yang telah memberikan kritikan dan saran sehingga makalah ini dapat diselesaikan dengan baik.

\section{Daftar Pustaka}

[1] Bartle, R.G., D.R Sherbert.1994. Introduction to Real Analysis. Second Edition. John Wiley dan Sons, Singapure.

[2] Chen, N., Xu, Z., Xia, M. 2013. Interval-valued hesitant preference relations and their applications to group decision making. Knowledge-Based Systems. 37: 528 $-540$

[3] Thakur, G.S., Thakur, R., Singh, R. 2014. New hesitant fuzzy opperators. Fuzzy Inf. Eng.. 6: 379 - 392

[4] Torra, V. 2010. Hesitant fuzzy sets. Int.J.Intell.Syst. 25(6): 529 - 539

[5] Torra, V., Narukawa, Y. On hesitant fuzzy sets and decision. 2009. In: Proceeding of the 18th IEEE International Conference on Fuzzy System, Jeju Island,pp. $1378-1382$

[6] Verma, R., Sharma, B.D. 2013. New operations over hesitant fuzzy sets. Fuzzy Inf. Eng. 2: $129-146$

[7] Xia, M., Xu,Z. 2011. Hesitant Fuzzy Information Aggregation in decision making. International Journal of Approximate Reasoning. 52: 395 - 407

[8] Xu, Z.S., Da, Q.L. 2002. The uncertain OWA operator. Int. J. Intell. Syst. 17: $569-575$

[9] Zadeh, L.A. 1965. Fuzzy sets. Inf. Control. 8: $338-353$ 